\title{
PALINOLOGIA DE DEPÓSITOS RUDÁCEOS DA REGIÃO DE ITATIAIA, BACIA DE RESENDE - RJ
}

\author{
Murilo Rodolfo de Lima* \& Mario Sergio de Melo**
}

\begin{abstract}
The Resende Basin is associated with rifting processes which characterize the Cenozoic geologic evolution of southeastern Brazil. Some palynological information is available from alluvial plain deposits of its sedimentary fill, the Resende Formation. Other associated deposits, having questionable relationships with this unit, have also been mentioned in the literature. One of these consists of rudaceous deposits of alluvial fans, in which the clasts are chiefly of alkaline rocks from the Itatiaia massif. These deposits, of controversed age, include silty-sand layers, very rich in organic matter, whose palynological potential motivated the research reported here.

The palynological association suggests an Oligocene age for the deposition of the organic matter. From a paleoecological point of view, this deposition took place under stagnant conditions, in a tropical to subtropical climate.

Geologically, these results reinforce the idea of a close genetic relationship between the rudaceous alluvial fan deposits and the alluvial plain sediments of the Resende Formation. It is proposed here the name of Itatiaia Formation for the alluvial fan deposits.

Palavras-chave: Pólens, Formação Itatiaia, Leques Aluviais, Rifte e Cenozóico
\end{abstract}

\section{INTRODUÇÃO}

A Bacia de Resende é uma depressão tectônica de idade terciária situada no médio curso do rio Paraíba do Sul, no extremo oeste do Estado do Rio de Janeiro. Apresenta forma alongada na direção N75E, com cerca de 50 quilômetros de comprimento e 5 a 6 quilômetros de largura, perfazendo área de 240 quilômetros quadrados de exposição de sedimentos terciários e quaternários. A espessura máxima dos sedimentos é hoje de cerca de 200 metros.

Tal depressão pertence ao "Sistema de Rifte da Serra do Mar" (ALMEIDA 1976), denominado "Rifte Continental do Sudeste do Brasil" (RICCOMINI 1989). Este sistema de rifte estende-se por 1000 quilômetros ao longo da faixa litorânea do sudeste do Brasil, compreendendo ainda as depressões de Curitiba, Sete Barras, São Paulo, Taubaté, Volta Redonda, Itaboraí, Guanabara e São João.

Os primeiros estudos detalhados sobre o preenchimento sedimentar da Bacia de Resende foram realizados por AMADOR (1975), que definiu as formações Resende (Mioceno/Plioceno) e Floriano (Pleistoceno), ambas constituídas por depósitos continentais, fluviais ou de cones aluviais coalescentes. A Formação Resende foi então subdividida em uma porção basal, arenosa a síltico-arenosa, de planície fluvial de rios anastomosados em clima semi-árido, e uma porção superior, rudácea, formada por corridas de lama sob clima mais úmido. Esta porção foi denominada "membro rudáceo da Formação Resende" (AMADOR 1975, p. 190), ocorrendo no flanco sul do maciço alcalino do Itatiaia.
Estudos do IPT (1983) subdividiram a Formação Resende em um membro rudáceo, constituído de brechas sedimentares e conglomerados de leques aluviais coalescentes, e uma porção fluvial, constituída de bancos alternados ou gradacionais de conglomerados a siltes argilosos. Consideraram as duas subdivisões como contemporâneas, interdigitadas. Admitiram idade eocena para a sedimentação, com base em correlação com os depósitos da Bacia de Volta Redonda, estes incluindo derrame de lavas ankaramíticas datados pelo método K-Ar. Proposta semelhante foi apresentada por MELO et al. (1985), que denominaram as subdivisões da Formação Resende fácies (rudácea e fluvial).

LIMA \& AMADOR (1985) realizaram estudo palinológico de sedimentos contendo níveis de linhitos provenientes da porção fluvial da Formação Resende. Admitiram idade eocena superior para a associação polínica encontrada, e interpretaram vigência de clima quente ou temperado, provavelmente úmido, durante a sedimentação.

RICCOMINI (1989) descreveu a Formação Resende incluindo depósitos de leques aluviais proximais (fácies com diamictitos e conglomerados) e de planície aluvial de rios entrelaçados e leques aluviais distais (fácies com arenitos, conglomerados e lamitos arenosos), contemporâneos e com transição lateral e interdigitações.

Como se nota, a tendência a partir da década de 80 tem sido de considerar os depósitos rudáceos da Bacia de Resende como resultado da sedimentação em leques aluviais proximais, contemporânea dos depósitos mais típicos de planície aluvial, desenvolvidos a jusante dos leques. 


\section{PALINOLOGIA}

Os sedimentos estudados por LIMA \& AMADOR (1985) são provenientes dos depósitos de planície aluvial de rios entrelaçados (local A da Fig. 1), comparáveis àqueles da seção-tipo da Formação Resende. Já os sedimentos analisados no presente estudo são provenientes de depósitos rudáceos típicos de leques aluviais proximais (local B da Fig. 1). As comparações entre as interpretações referentes à cronologia e paleoclimas dos sedimentos estudados permitem aperfeiçoar as interpretações envolvendo o preenchimento da Bacia de Resende e a proposição de coluna estratigráfica adequada.

\section{PROVENIÊNCIA DA AMOSTRA ESTUDADA}

A amostra estudada no presente trabalho provém de depósitos rudáceos da Bacia de Resende, expostos em afloramento (grande corte em jazida de materiais de empréstimo e de construção) situado junto à ferrovia (antiga EFCB) na área urbana de Itatiaia, do lado esquerdo da subida de acesso ao Parque Nacional do Itatiaia (local B da Fig. 1).

Os depósitos rudáceos são ali constituídos predominantemente por diamictitos em corpos com espessura de até 2 metros, com intercalações menores de conglomerados, areias conglomeráticas e lentes com espessura decimétrica de silte-arenoso com restos de matéria orgânica (inclusive alguns moldes de estruturas vegetais), onde foi coletada a amostra para estudo palinológico (Fig. 2). Situados junto ao flanco sul do maciço alcalino do Itatiaia, os depósitos apresentam, em geral, clastos constituídos de rochas alcalinas, sendo raros os clastos de rochas do embasamento cristalino.

Estes depósitos rudáceos são típicos de leques aluviais proximais, com predominância de processos de corridas de lamas, formados junto à borda norte da Bacia de Resende, quando de paleo-relevo escarpado, pela presença de falhas normais e do maciço do Itatiaia.

Topograficamente, o afloramento estudado por LIMA \& AMADOR (1985) situa-se aproximadamente na mesma cota do afloramento estudado neste trabalho; a distância entre eles é de 3,5 quilômetros (Fig. 1). Nada se pode afirmar sobre a posição estratigráfica relativa entre os dois afloramentos, salvo que eles representam depósitos de leques aluviais proximais (analisados no presente estudo) que passam lateralmente para depósitos de planície aluvial (analisados por LIMA \& AMADOR 1985).

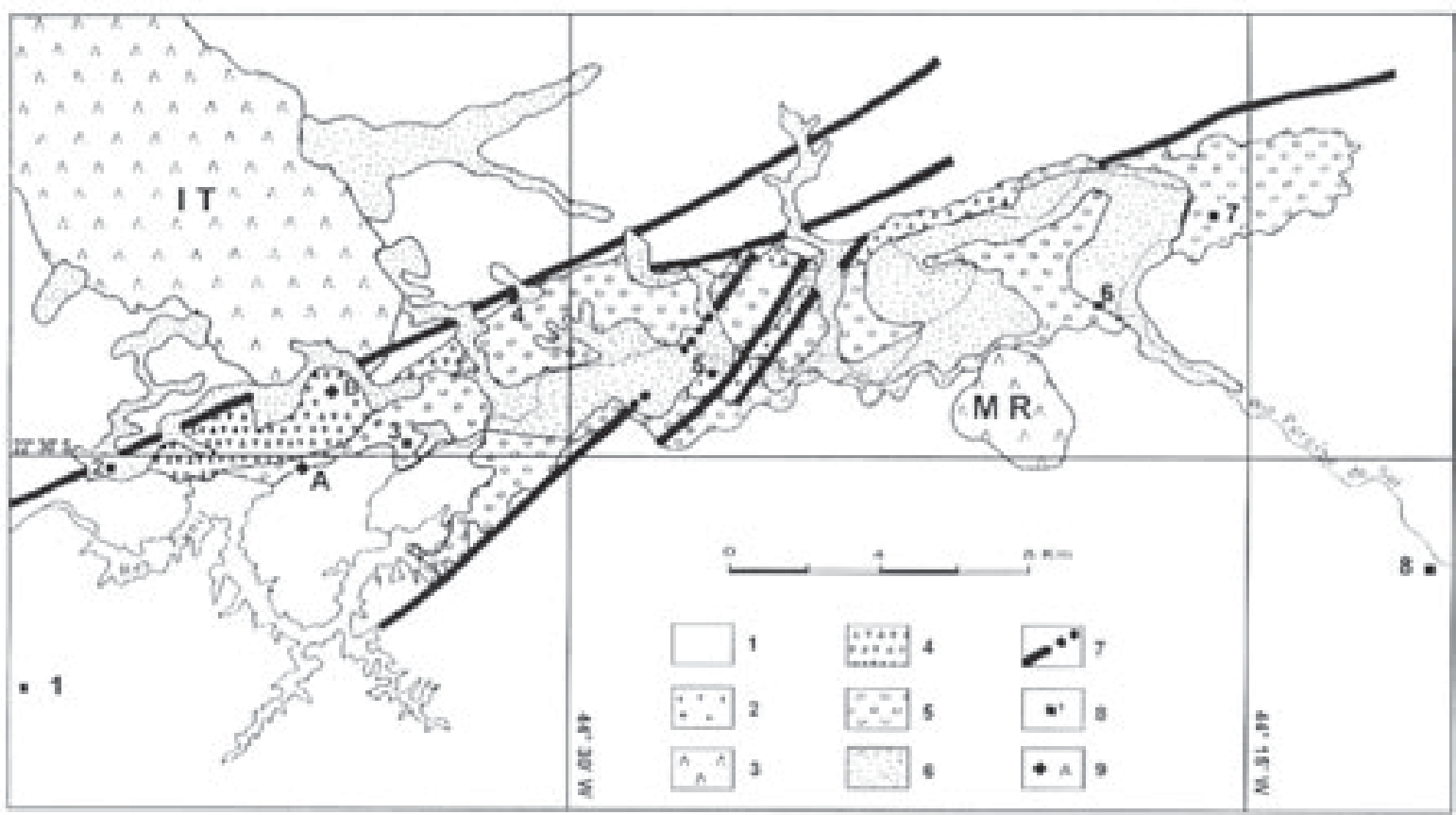

Figura 1 - Esboço geológico da Bacia de Resende (baseado em MELO et al. 1985 e RICCOMINI 1989). 1: embasamento pré-cambriano; 2: afloramento do embasamento no alto estrutural de Resende; 3: maciços alcalinos do Itatiaia (IT) e Morro Redondo (MR); 4: sedimentos terciários rudáceos de leques aluviais

(Formação Itatiaia); 5: sedimentos terciários de planície aluvial, atribuíveis às formações Resende e São

Paulo; 6: sedimentos quaternários; 7: principais falhas; 8: localidades (1=Areias, 2=Engenheiro Passos, $3=$ Itatiaia, 4=Penedo, $5=$ Resende, $6=$ Floriano, $7=$ Quatis, $8=$ Barra Mansa); 9: local do afloramento estudado por LIMA \& AMADOR 1985 (A) e local do afloramento da Figura 2, estudado neste trabalho (B).

Figure 1 - Geological sketch map of the Resende Basin (after MELO et al. 1985 and RICCOMINI 1989).

1 - precambrian basement; 2: basement outcrop in the Resende uplift; 3: alcaline complexes: Itatiaia (IT) and Morro Redondo (MR); 4: tertiary sediments: alluvial fan gravels (Itatiaia Formation); 5: tertiary sediments: alluvial flood plain, correlated with Resende and São Paulo Formations. 6: quaternary sediments; 7: principal

faults; 8: localities (1=Areias, 2=Engenheiro Passos, 3=Itatiaia, 4=Penedo, 5=Resende, $6=$ Floriano,

7=Quatis, 8=Barra Mansa); 9: location of the outcrop studied by LIMA \& AMADOR 1985 (A) and of the outcrop of Figure 2 (this paper) (B). 


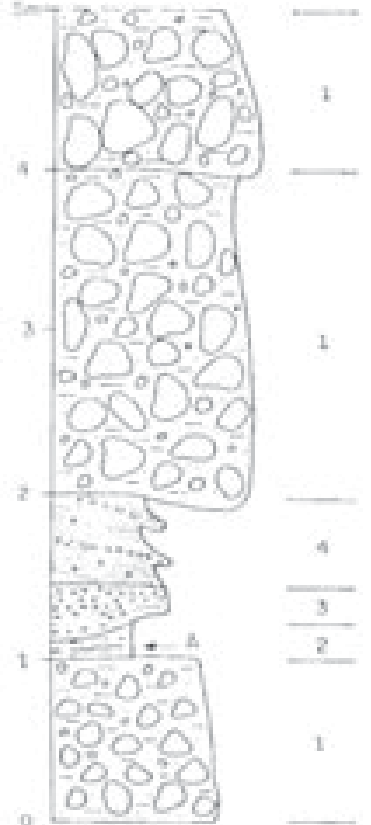

Figura 2 - perfil esquemático da parte basal do afloramento de sedimentos terciários rudáceos de leques aluviais (Formação Itatiaia) da Bacia de Resende (localização na Figura 1). 1: diamictitos; 2. silte-arenoso com restos de matéria orgânica ( $A=$ local de coleta da amostra com conteúdo palinológico aqui estudado); 3: conglomerado fino; 4: areia conglomerática.

Figure 2 - Schematic section of the basal part of the alluvial fan gravel of the Resende Basin (Tertiary,

Itatitaia Formation; for location see Figure 1). 1: diamictites; 2: arenaceous silt with organic matter (A = sample site of this study); 3: small pebble conglomerate; 4 : pebbly sand.

\section{Classificação}

Do material coletado foi processada uma amostra, segundo técnica palinológica padrão. Dela, duas lâminas foram montadas, estando depositadas na coleção científica do Departamento de Paleontologia e Estratigrafia do IG-USP sob os números GP/4T-327 e GP/4T-328.

O material é fértil e bastante diversificado, compondo-se de fungos, algas, esporos e grãos de pólen de gimnospermas e angiospermas. Quarenta e quatro espécies (à exceção dos fungos, que serão objeto de trabalho especial) foram identificadas. A lista completa destes táxons é a seguinte:

\section{ALGAE}

Gênero Pseudoschizaea THIEGART \& FRANTZ 1962

-Pseudoschizaea circula (WOLF) CRISTOPHER

Estampa I, fig. 1

Afinidade botânica: indeterminada

\section{SPORITES}

Turma Triletes (REINSCH 1881) POTONIÉ \& KREMP 1954
Subturma Azonotriletes LUBER 1936 emend. DETTMANN 1963

Infraturma Laevigati BENNIE \& KIDSTON 1886

Gênero Stereisporites PFLUG 1953

-Stereisporites psilatus (ROSS) MANUM

Estampa I, fig. 2

Afinidade botânica: Sphagnaceae

Gênero Leiotriletes (NAUMOVA 1937) POTONIÉ \& KREMP 1954

-Leiotriletes adriennis KRUTZSCH

Estampa I, fig. 3

Afinidade botânica: Pteridophyta

Gênero Deltoidospora MINER 1935 emend. POTONIÉ 1956

-Deltoidospora minor (COUPER) POCOCK

Estampa I, fig. 4

Afinidade botânica: Lindsaya

Gênero Todisporites COUPER 1958

-Todisporites sp.

Estampa I, fig. 5

Afinidade botânica: Osmundaceae

Infraturma Murornati POTONIÉ \& KREMP 1954

Gênero Cicatricosisporites POTONIÉ \& GELLETICH 1933

-Cicatricosisporites dorogensis POTONIÉ \& GELLETICH

Estampa I, figs. 6-7

Afinidade botânica: Schizeaceae

-Cicatricosisporites cristatus REGALI et al.

Estampa I, fig. 8

Afinidade botânica: Schizeaceae

-Cicatricosisporites newmani BOLTENHAGEN

Estampa I, fig. 9

Afinidade botânica: Schizeaceae

-Cicatricosisporites sp.

Estampa I, fig. 10

Afinidade botânica: Schizeaceae

Gênero Magnastriatites GERMERAAD et al. 1968

-Magnastriatites howardi GERMERAAD et al.

Estampa I, fig. 11

Afinidade botânica: Schizeaceae

Turma Zonales (BENNIE \& KIDSTON 1886)

POTONIÉ 1956

Subturma Zonotriletes WALTZ 1935

Infraturma Cingulati POTONIÉ \& KLAUS 1954

Gênero Polypodiaceoisporites POTONIÉ 1956

-Polypodiaceoisporites potoniei KEDVES

Estampa I, fig. 12

Afinidade botânica: Polypodiaceae

Turma Monoletes IBRAHIM 1933

Subturma Azonomonoletes LUBER 1935

Infraturma Laevigatomonoleti DYBOWA \& JACHOWICZ 1957 
Gênero Laevigatosporites IBRAHIM 1933

-Laevigatosporites ovatus WILSON \& WEBSTER

Estampa I, fig. 13

Afinidade botânica: Polypodiaceae

-Laevigatosporites haardti (POTONIÉ \& VENKATACHALA) THOMSON \& PFLUG

Estampa I, fig. 14

Afinidade botânica: Polypodiaceae

Gênero Punctatosporites IBRAHIM 1933

-Punctatosporites sp.

Estampa I, figs. 16-17

Afinidade botânica: Polypodiaceae

Infraturma Sculptatomonoleti DYBOWA \& JACHOWICZ 1957

Gênero Verrucatosporites PFLUG 1952

-Verrucatosporites tennelis KRUTZSCH

Estampa I, fig. 15

Afinidade botânica: Polypodiaceae

-Verrucatosporites balticus KRUTZSCH

Estampa I, fig. 18

Afinidade botânica: Polypodiaceae

-Verrucatosporites rugufavus KRUTZSCH

Estampa I, fig. 19

Afinidade botânica: Polypodiaceae

\section{POLLENITES}

Turma Saccites ERDTMAN 1947

Subturma Disaccites COOKSON 1947

Gênero Pityosporites SEWARD 1914

-Pityosporites microalatus (POTONIÉ)

THOMSON \& PFLUG

Estampa II, fig. 1

Afinidade botânica: Podocarpaceae

Gênero Podocarpidites COOKSON 1947

-Podocarpidites ellipticus COOKSON

Estampa II, fig. 2

Afinidade botânica: Podocarpaceae

Turma Plicates NAUMOVA 1937-1939

Subturma Monocolpates IVERSEN \& TROELSSMITH 1950

Gênero Psilamonocolpites MATHUR 1966

-Psilamonocolpites sp.

Estampa II, fig. 3

Afinidade botânica: Arecaceae

Subturma Tricolpates IVERSEN \& TROELS-

SMITH 1950

Gênero Scabratricolpites (VAN DER HAMMEN 1956) GONZÁLEZ-GUZMÁN 1967

-Scabratricolpites circulus CHATEAUNEUF

Estampa II, fig. 4

Afinidade botânica: Dycotiledoneae

Turma Poroses (NAUMOVA 1939) POTONIÉ 1960

Subturma Triporates IVERSEN \& TROELSSMITH 1950
Gênero Proteacidites COOKSON 1950

-Proteacidites dehaani GERMERAAD et al.

Estampa II, fig. 6

Afinidade botânica: Proteaceae

-Proteacidites rectomarginatus COOKSON

Estampa II, fig. 5

Afinidade botânica: Proteaceae

Subturma Stephanoporates IVERSEN \& TROELSSMITH 1950

Gênero Ulmoideipites ANDERSON 1960

-Ulmoideipites krempii ANDERSON

Estampa II, fig. 7

Afinidade botânica: Ulmaceae

Gênero Miocaenipollis KRUTZSCH

-Miocaenipollis miocaenicus KRUTZSCH

Estampa II, figs. 8 e 10

Afinidade botância: Malpighiaceae?

-Miocaenipollis sp.

Estampa II, fig. 9

Afinidade botânica: Malpighiaceae?

Gênero Echiperiporites VAN DER HAMMEN \& WIJMSTRA 1964

-Echiperiporites akanthos VAN DER HAMMEN

\& WIJMSTRA

Estampa II, fig. 16

Afinidade botânica: Dicotyledoneae

Gênero Catinipollis KRUTZSCH 1966

-Catinipollis geiseltalensis KRUTZSCH

Estampa II, fig. 15

Afinidade botânica: Craniolaria (Martyniaceae)

Gênero Parsonsidites COUPER 1960

-Parsonsidites sp. cf. P. psilatus COUPER

Estampa II, fig. 11

Afinidade botânica: Apocynaceae

Observações: os dois exemplares encontrados apresentam um elevado grau de corrosão; os caracteres diagnósticos permitem, contudo, uma identificação satisfatória.

Gênero Psilaperiporites REGALI et al. 1974

-Psilaperiporites minimus REGALI et al.

Estampa II, fig. 14

Afinidade botânica: Chenopodiaceae

Gênero Psilastephanoporites (VAN DER HAMMEN 1956) REGALI et al. 1974

-Psilastephanoporites stellatus REGALI et al.

Estampa II, figs. 12-13

Afinidade botânica: Malpighiaceae

Subturma Tricolporates IVERSEN \& TROELSSMITH 1950

Gênero Psilatricolporites (VAN DER HAMMEN 1956) VAN DER HAMMEN \& WIJMSTRA 1964

-Psilatricolporites costatus DUEÑAS 
Estampa II, figs 17-18

Afinidade botânica: Dicotyledoneae

-Psilatricolporites cyamus VAN DER HAMMEN

\& WIJMSTRA

Estampa II, fig. 19

Afinidade botânica: Dicotyledoneae

Gênero Scabratricolporites (VAN DER HAMMEN 1956) RAMANUJAM 1966

-Scabratricolporites sp.

Estampa II, fig. 20

Afinidade botânica: Dicotyledoneae

Gênero Foveotricolporites PIERCE 1961

-Foveotricolporites $\mathrm{sp}$.

Estampa II, fig. 22

Afinidade botânica: Dicotyledoneae

\section{Gênero Echitricolporites VAN DER HAMMEN} 1956

-Echitricolporites spinosus VAN DER HAMMEN

Estampa II, fig. 24

Afinidade botânica: Compositae?

Gênero Margocolporites RAMANUJAM 1966

-Margocolporites cribellatus SRIVASTAVA

Estampa II, fig. 23

Afinidade botânica: Concolvulaceae

Subturma Stephanocolporates IVERSEN \& TROELS-SMITH 1950

\section{Gênero Psilastephanocolporites \\ LEIDELMEYER 1966}

-Psilastephanocolporites fissilis LEIDELMEYER

Estampa II, fig. 25

Afinidade botânica: Polygalaceae

-Psilastephanocolporites sp. cf.P. minor SALARD-

CHEBOLDAEFF

Estampa II, fig. 21

Afinidade botânica: Dicotyledoneae

-Psilastephanocolporites sp.

Estampa II, fig. 27

Afinidade botânica: Dicotyledoneae

Subturma Syncolporites IVERSEN \& TROELS-

SMITH 1950

Gênero Syncolporites VAN DER HAMMEN 1954 -Syncolporites poricostatus VAN HOEKENKLINKENBERG

Estampa II, fig. 26

Afinidade botânica: Myrtaceae

\section{Gênero Perisyncolporites GERMERAAD et al. 1968}

-Perisyncolporites pokornyi GERMERAAD et al. Estampa II, figs. 28-29

Afinidade botânica: Malpighiaceae

Turma Jugates ERDTMAN 1947

Subturma Polyadites PANT 1954
Gênero Acaciapollenites MILDENHALL 1972

-Acaciapollenites myriosporites (COOKSON 1954)

Estampa II, fig. 30

Afinidade botânica: Acacia

\section{INCERTAE SEDIS}

\section{Gênero Incrotonipollis JANSONIUS \& HILLS 1981}

-Incrotonipollis sp.

Estampa II, fig. 33

Afinidade botânica: Dicotyledoneae

Observações: exemplares em tudo idênticos aos referidos para a Bacia do Macacu, Terciário do Estado do Rio de Janeiro.

\section{OUTROS RESTOS VEGETAIS}

Tricoma tipo 1 (simples)

Estampa II, fig. 31

Afinidade botânica: Violaceae?

Tricoma tipo 2 (estrelado)

Estampa II, fig. 32

Afinidade botânica: Malvaceae

\section{DISCUSSÃO}

A associação polínica encontrada permite discutir aspectos relacionados com a idade, correlações com outras bacias do sudeste do Brasil, paleoambiente e paleoclima.

Idade

Até o presente estudo, a idade dos depósitos rudáceos aqui estudado não tinha sido confirmada. A fácies sedimentar representada pelo afloramento estudado é clássica na região, e já tem sido objeto de considerações na literatura, como as efetuadas por AMADOR (1975), que a denominou "membro rudáceo da Formação Resende", e a considerou como fecho da deposição desta unidade. Em trabalhos posteriores (AMADOR \& BREHME 1978, AMADOR et al. 1978) este ponto de vista foi reafirmado, e a seqüência correspondente considerada pliocena, a partir de critérios geomorfológicos.

LIMA \& AMADOR (1985) introduziram os dados palinológicos no conhecimento da bacia, datando sedimentos de planície aluvial da Formação Resende como sendo do Eoceno, e situando-os no contexto do "Sistema de Rifte da Serra do Mar".

No estudo atual, onde os sedimentos rudáceos são analisados, os resultados obtidos são coerentes com as interpretações relativas ao relacionamento entre estes e os sedimentos de planície aluvial da Formação Resende.

Entre as diversas espécies de palinomorfos presentes, algumas merecem atenção especial por constituírem guias estratigráficos em utilização no Brasil. É o caso de Cicatricosisporites dorogensis, 
Cicatricosisporites cristatus, Proteacidites dehaani, Catinipollis geiseltalensis, Magnastriatites howardi e Psilastephanoporites stellatus. Destas, as duas últimas sugerem fortemente idade oligocena para os níveis portadores, distinta, portanto, apesar de próxima, da atribuída para os depósitos de planície aluvial da Formação Resende.

\section{Correlações}

Várias das espécies presentes já foram registradas para outras bacias do sudeste brasileiro. A Fig. 3 ilustra o registro destes táxons na região, sendo baseada na literatura existente. No caso específico de REGALI et al. (1974a e 1974b), a Bacia de Campos foi interpretada como equivalente à Plataforma Continental do Rio de Janeiro, e a de Santos à Plataforma Continental de São Paulo.

Como pode ser verificado na Fig. 3, a Bacia de Resende apresenta maior semelhança com a Bacia de Macacu, com 14 espécies comuns. Deve ser mencionado que apenas os táxons classificados a nível específico foram considerados nesta comparação, sendo possível que a identidade de formas seja algo maior.

\section{Paleoambiente e Paleoclima}

Elementos do microplâncton marinho estão completamente ausentes na associação, corroborando origem continental para os sedimentos estudados. A enorme abundância e diversidade de fungos aponta para condições estagnantes, num corpo aquoso provavelmente pequeno e efêmero dentro do sistema de leques aluviais.

A abundância de fungos e esporos pressupõe um clima tropical a subtropical úmido durante a deposição deste pacote. Proteáceas, mirtáceas, leguminosas e poligaláceas reforçam esta conclusão. Aspecto interessante diz respeito à presença de tricomas, tanto simples como estrelados, os primeiros interpretados como pêlos de violáceas e os segundos de malváceas.

\section{CONSIDERAÇÕES FINAIS}

A idade obtida para o sedimento estudado, embora não corresponda exatamente àquela divulgada por LIMA \& AMADOR (1985), situa a sedimentação no Paleógeno, reforçando a idéia de relações genéticas estreitas entre os depósitos rudáceos de leques aluviais ora estudados e os depósitos de planície aluvial da Formação Resende, conforme vem sendo admitido nos estudos realizados a partir da década de 80 .

Os paleoclimas interpretados também são semelhantes, apontando para condições quentes a temperadas e úmidas para os dois tipos de sistemas deposicionais. Isto constitui argumento adicional para a idéia de trato deposicional, sincrônico, abrangendo os leques e a planície aluvial.

As diferenças litológicas entre os depósitos rudáceos de leques aluviais e os depósitos mais finos de planície aluvial de rios entrelaçados, bem como as relações de contato e geometria, que permitem separar esses depósitos em mapa, apontam para a conveniência prática de distingui-los na proposição de coluna estratigráfica para a Bacia de Resende.

Propõe-se aqui designar os depósitos rudáceos de leques aluviais de Formação Itatiaia, e indica-se o afloramento da Fig. 2 como seção-tipo. A designação Formação Resende ficaria, assim, restrita aos depósitos mais finos, de planície aluvial de rios entrelaçados, ocasionalmente incluindo lamitos arenosos de porções distais de leques aluviais. Oportunamente, será apresentado trabalho detalhando características litológicas e geométricas da Formação Itatiaia.

\section{AGRADECIMENTOS}

Os autores agradecem ao Prof. Dr. Thomas Rich Fairchild pela leitura crítica do abstract, bem como à Universidade de Guarulhos, na pessoa da Profa. Maria Judite Garcia, pelo uso do fotomicroscópio.

\section{REFERÊNCIAS BIBLIOGRÁFICAS}

ALMEIDA, F.F.M. de. 1976. The system of continental rifts bordering the Santos Basin. An. Acad. Bras. Ciênc., v.48 (supl.), p.13-26. AMADOR, E. da S. 1975. Estratigrafia e sedimentação da Bacia de Resende, R.J. An. Acad. bras. Ciênc., vol.47, p.181-225. (Suplemento).

AMADOR, E. da S. \& BREHME, I. 1978. Significado paleoambiental dos rudáceos cenozóicos da Bacia de Resende. An. Acad. bras. Ciênc., v.50, n.1, p.123 (Resumo).

AMADOR, E. da S., ANTUNES, R.L., PAIXÃO, R.A. 1978. Notas complementares sobre a estratigrafia dos depósitos cenozóicos da Bacia de Resende. An. Acad. bras. Ciênc., v.50, n.1, p.122123 (Resumo).

INSTITUTO DE PESQUISAS TECNOLÓGICAS DO ESTADO DE SÃO PAULO - IPT. 1983. Estudos geológico-tectônicos na Bacia de Resende (RJ) e sedimentos terciários da área de Volta Redonda (RJ) e Bacia de Taubaté (área de Cruzeiro, SP). São Paulo. (IPT, Relatório 17 737).

LIMA, M.R. de \& AMADOR, E. da S. 1985. Análise palinológica de sedimentos da Formação Resende, Terciário do Estado do Rio de Janeiro, Brasil. In: Coletânea de trabalhos paleontológicos, Brasília, DNPM. Série Geologia, n.27, p.371-378. (Seção Paleontologia e Estratigrafia, n.2).

LIMA, M.R. de \& ÂNGULO, R.J. 1990. Descoberta de microflora em um nível linhítico da Formação Alexandra, Terciário do Estado do Paraná, Brasil.An. Acad. bras. Ciênc., v.62, n.4, p.357371.

LIMA, M.R. de \& CUNHA, F.L.S. 1986. Análise palinológica de um nível linhítico da Bacia de São José de Itaboraí, Terciário do Estado do Rio de Janeiro, Brasil. An. Acad. bras. Ciênc., v.58, n.4, p.579-588.

LIMA, M.R. de \& DINO, R. 1984. Palinologia de amostras da Bacia de Bonfim, Terciário do Estado de São Paulo, Brasil. Bol. IGUSP. Série Cient. n.15, p.1-11.

LIMA, M.R. de \& SALARD-CHEBOLDAEFF, M. 1981. Palynologie des bassins de Gandarela et Fonseca (Eocène de l'État de Minas Gerais, Brésil). Bol. IGUSP, Série Cient. n.12, p.33-54.

LIMA, M.R. de, SALARD-CHEBOLDAEFF, M., SUGUIO, K. 1985. Étude palynologique de la Formation Tremembé, Tertiaire du Bassin de Taubaté (État de São Paulo, Brésil), d'après les échantillons du sondage ${ }^{\circ} 42$ du CNP. In: Coletânea de trabalhos paleontológicos, Brasília, DNPM. Série Geologia n²7, p.379393. (Seção Paleontologia e Estratigrafia, n.2). 


\begin{tabular}{|c|c|c|c|c|c|c|c|c|c|c|c|c|c|}
\hline Pseudoschizaea circula & & & & & & $\bullet$ & & & $\bullet$ & & $\bullet$ & & \\
\hline Stereisporites psilatus & & & & & & & & & & & & & \\
\hline Leiotriletes adriennis & & & & & & & $\bullet$ & $\bullet$ & & & $\bullet$ & & \\
\hline Deltoidospora minor & & & & & & $\bullet$ & & & & & & & \\
\hline Cicatricosisporites dorogensis & & $\bullet$ & - & $\bullet$ & $\bullet$ & $\bullet$ & $\bullet$ & $\bullet$ & $\bullet$ & $\bullet$ & & $\bullet$ & • \\
\hline Cicatricosisporites cristatus & & & & & & & & & & & & $\bullet$ & \\
\hline Cicatricosisporites newmani & & & & $\bullet$ & & & & & & & & & \\
\hline Magnastriatites howardi & & & & & & & & & & & & & \\
\hline Polypodiaceoisporites potoniei & & & & $\bullet$ & & $\bullet$ & $\bullet$ & $\bullet$ & & $\bullet$ & & $\bullet$ & $\bullet$ \\
\hline Laevigatosporites ovatus & & & $\bullet$ & $\bullet$ & & $\bullet$ & $\bullet$ & $\bullet$ & $\bullet$ & $\bullet$ & $\bullet$ & & \\
\hline Laevigatosporites haardti & & $\bullet$ & & & & & & & & & & & \\
\hline Verrucatosporites tennelis & & & $\bullet$ & & & & $\bullet$ & & $\bullet$ & & & & \\
\hline Verrucatosporites balticus & & & & & & & & & & & & & \\
\hline Verrucatosporites rugufavus & & & & & & & & & & & & & \\
\hline Pityosporites microalatus & & & & & & & & & $\bullet$ & $\bullet$ & & & \\
\hline Podocarpidites ellipticus & & & & $\bullet$ & $\bullet$ & $\bullet$ & & & & & & & \\
\hline Scabratricolpites circulus & & & & & & & & & & & & & \\
\hline Proteacidites rectomarginatus & & & & & & $\bullet$ & & & $\bullet$ & & & & \\
\hline Proteacidites dehaani & & & & & & $\bullet$ & $\bullet$ & $\bullet$ & $\bullet$ & & & & \\
\hline Ulmoideipites krempii & $\bullet$ & & - & - & $\bullet$ & $\bullet$ & $\bullet$ & - & - & $\bullet$ & & $\bullet$ & $\bullet$ \\
\hline Miocaenipollis miocaenicus & & & & $\bullet$ & & $\bullet$ & $\bullet$ & $\bullet$ & & & $\bullet$ & & \\
\hline Parsonsidites sp.cf.P.psilatus & & $\bullet$ & & & & & & $\bullet$ & & & & & \\
\hline Psilastephanoporites stellatus & & & & & & & $\bullet$ & & & $\bullet$ & & & \\
\hline Psilaperiporites minimus & & & & & & & $\bullet$ & & & $\bullet$ & & & \\
\hline Catinipollis geiseltalensis & & & & $\bullet$ & $\bullet$ & & $\bullet$ & $\bullet$ & $\bullet$ & $\bullet$ & & & \\
\hline Echiperiporites akanthos & $\bullet$ & & $\bullet$ & $\bullet$ & $\bullet$ & $\bullet$ & & & & $\bullet$ & & $\bullet$ & $\bullet$ \\
\hline Psilatricolporites costatus & & & & & & & & $\bullet$ & & & & & \\
\hline Psilatricolporites cyamus & & & & & & $\bullet$ & & & & & & & \\
\hline Margocolporites cribellatus & & & & & & & & $\bullet$ & & & & & \\
\hline Echitricolporites spinosus & & & & & & & & & & & & $\bullet$ & $\bullet$ \\
\hline Psilastephanocolporites fissilis & & $\bullet$ & $\bullet$ & & $\bullet$ & & $\bullet$ & & & & & $\bullet$ & $\bullet$ \\
\hline Psilastephanocolporites sp.cf.P.minor & & & & & $\bullet$ & & & & & & & & \\
\hline Syncolporites poricostatus & & & $\bullet$ & & & & & & $\bullet$ & & & $\bullet$ & $\bullet$ \\
\hline Perisyncolporites pokornyi & & & $\bullet$ & $\bullet$ & & $\bullet$ & $\bullet$ & - & $\bullet$ & $\bullet$ & $\bullet$ & $\bullet$ & $\bullet$ \\
\hline Acaciapollenites myriosporites & & & & & & $\bullet$ & $\bullet$ & & & & & & \\
\hline $\begin{array}{l}\text { Observação: Dados extraídos de Lima \& Amador } \\
\text { (1985), Lima \& Angulo (1990), Lima \& Cunha (1986), } \\
\text { Lima \& Dino (1984), Lima \& Salard-Cheboldaeff } \\
\text { (1981), Lima et al.(vários), Melo et al. 1985, Pinto e } \\
\text { Regali (1991) e Regali et al. (1974 a, b). }\end{array}$ & 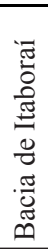 & 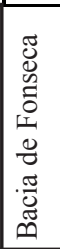 & 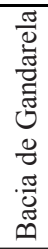 & 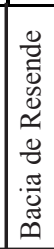 & 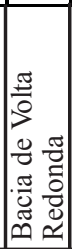 & 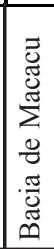 & 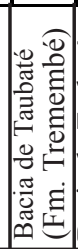 & 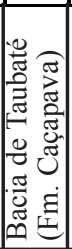 & 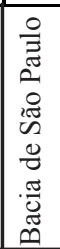 & 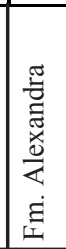 & 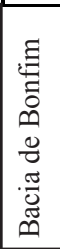 & 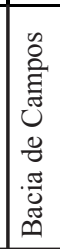 & 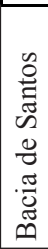 \\
\hline
\end{tabular}


LIMA, M.R. de, VESPUCCI, J.B.O., SUGUIO,K. 1985. Estudo palinológico de uma camada de linhito da Formação Caçapava, Bacia de Taubaté, Terciário do Estado de São Paulo, Brasil. An. Acad. bras. Ciênc., v.57, n.2, p.183-197.

LIMA, M.R. de, MELO, M.S. de, COIMBRA, A.M. 1991. Palinologia de sedimentos da Bacia de São Paulo, Terciário do Estado de São Paulo, Brasil. Rev. $I G$, São Paulo, v.12, n.1/2, p.7-20.

LIMA, M.R. de, RICCOMINI, C., SOUZA, P.A. 1992. Palinologia de folhelhos do gráben de Casa de Pedra, Terciário do Estado do Rio de Janeiro, Brasil. In: CONGRESSO BRASILEIRO DE PALEONTOLOGIA, 13, São Leopoldo, 1992. Resumos... SBP, p.163.

LIMA, M.R. de, CABRAL JR., M., STEFANI, F.L. s.d. Palinologia de sedimentos da Formação Macacu - Rifte da Guanabara, Terciário do Estado do Rio de Janeiro, Brasil. An. Acad. bras. Ciênc. (no prelo).
MELO, M.S. de, VINCENS, A., TUCHOLKA, P. 1985. Contribuição à cronologia da Formação Itaqua-quecetuba - SP. An. Acad. bras. Ciênc., v.57, n.2, p.175-181.

MELO, M.S. de, RICCOMINI, C., ALMEIDA, F.F.M. de, HASUI, Y. 1985. Sedimentação e tectônica da Bacia de Resende, RJ. An. Acad. bras. Ciênc., v.57, n.4, p.467-479.

PINTO, A.D.P. \& REGALI, M.S.P. 1991. Palin-oestratigrafia dos sedimentos terciários da Bacia de Gandarela, Minas Gerais, Brasil. Rev. Esc. Minas Ouro Preto, v.44, n.1, p.10-15.

REGALI, M.S.P., UESUGUI, N., SANTOS, A.S. 1974a. Palinologia dos sedimentos meso-ceno-zóicos do Brasil I. Bol. Técn. Petrobrás, v.17, n.3, p.177-191.

REGALI, M.S.P., UESUGUI, N., SANTOS, A.S. 1974b. Palinologia dos sedimentos meso-ceno-zóicos do Brasil II. Bol. Técn. Petrobrás, v.17, n.4, p.263-301.

RICCOMINI, C. 1989. O rift continental do Sudeste do Brasil. São Paulo. 256p. (Tese de Doutoramento, IGUSP).

\section{ESTAMPA I}

Obs.: todas as figuras aumentadas $800 \mathrm{X}$

Fig. 1 - Pseudoschizaea circula. Lâm. GP/4T-327.

Fig. 2 - Stereisporites psilatus. Lâm. GP/4T-327.

Fig. 3 - Leiotriletes adriennis. Lâm. GP/4T-327.

Fig. 4 - Deltoidospora minor. Lâm. GP/4T-327.

Fig. 5 - Todisporites sp. Lâm. GP/4T-327.

Fig. 6 - Cicatricosisporites dorogensis. Lâm. GP/4T-328.

Fig. 7 - Cicatricosisporites dorogensis. Lâm. GP/4T-328.

Fig. 8 - Cicatricosisporites cristatus. Lâm. GP/4T-327.

Fig. 9 - Cicatricosisporites newmani. Lâm. GP/4T-327.

Fig. 10 - Cicatricosisporites sp. Lâm. GP/4T-327.

Fig. 11 - Magnastriatites howardi. Lâm. GP/4T-328.

Fig. 12 - Polypodiaceoisporites potoniei. Lâm. GP/4T-328.

Fig. 13 - Laevigatosporites ovatus. Lâm. GP/4T-327.

Fig. 14 - Laevigatosporites haardti. Lâm. GP/4T-327.

Fig. 15 - Verrucatosporites tennelis. Lâm. GP/4T-327.

Fig. 16 - Punctatosporites sp. Lâm. GP/4T-328.

Fig. 17 - Punctatosporites sp. Lâm. GP/4T-328.

Fig. 18 - Verrucatosporites balticus. Lâm. GP/4T-328.

Fig. 19 - Verrucatosporites rugufavus. Lâm. GP/4T-328.

\section{ESTAMPA II}

Obs.: todas as figuras aumentadas $800 \mathrm{X}$

Fig. 1 - Pityosporites microalatus. Lâm. GP/4T-327.

Fig. 2 - Podocarpidites ellipticus. Lâm. GP/4T-328.

Fig. 3 - Psilamonocolpites sp. Lâm. GP/4T-327.

Fig. 4 - Scabratricolpites circulus. Lâm. GP/4T-327.

Fig. 5 - Proteacidites rectomarginatus. Lâm. GP/4T-328.
Fig. 6 - Proteacidites dehaani. Lâm. GP/4T-328.

Fig. 7 - Ulmoideipites krempii. Lâm. GP/4T-327.

Fig. 8 - Miocaenipollis miocaenicus. Lâm. GP/4T-328.

Fig. 9 - Miocaenipollis sp. Lâm. GP/4T-328.

Fig. 10 - Miocaenipollis miocaenicus. Lâm. GP/4T-327.

Fig. 11 - Parsonsidites sp. cf. P. psilatus. Lâm. GP/4T-328.

Fig. 12 - Psilastephanoporites stellatus. Lâm. GP/4T-327.

Fig. 13 - Psilastephanoporites stellatus. Lâm. GP/4T-327.

Fig. 14 - Psilaperiporites minimus. Lâm. GP/4T-328.

Fig. 15 - Catinipollis geiseltalensis. Lâm. GP/4T-327.

Fig. 16 - Echiperiporites akanthos. Lâm. GP/4T-327.

Fig. 17 - Psilatricolporites costatus. Lâm. GP/4T-327.

Fig. 18 - Psilatricolporites costatus. Lâm. GP/4T-328.

Fig. 19 - Psilatricolporites cyamus. Lâm. GP/4T-327.

Fig. 20 - Scabratricolporites sp. Lâm. GP/4T-327.

Fig. 21 - Psilastephanocolporites sp. cf. P. minor. Lâm. GP/ 4T-327.

Fig. 22 - Foveotricolporites sp. Lâm. GP/4T-328.

Fig. 23 - Margocolporites cribellatus. Lâm. GP/4T-327.

Fig. 24 - Echitricolporites spinosus. Lâm. GP/4T-327.

Fig. 25 - Psilastephanocolporites fissilis. Lâm. GP/4T-327.

Fig. 26 - Syncolporites poricostatus. Lâm. GP/4T-328.

Fig. 27 - Psilastephanocolporites sp. Lâm. GP/4T-327.

Fig. 28 - Perisyncolporites pokornyi. Lâm. GP/4T-327.

Fig. 29 - Perisyncolporites pokornyi. Lâm. GP/4T-327.

Fig. 30 - Acaciapollenites myriosporites. Lâm. GP/4T-328.

Fig. 31 - Tricoma simples. Lâm. GP/4T-327.

Fig. 32 - Tricoma estrelado. Lâm. GP/4T-328.

Fig. 33 - Incrotonipollis sp. Lâm. GP/4T-328. 

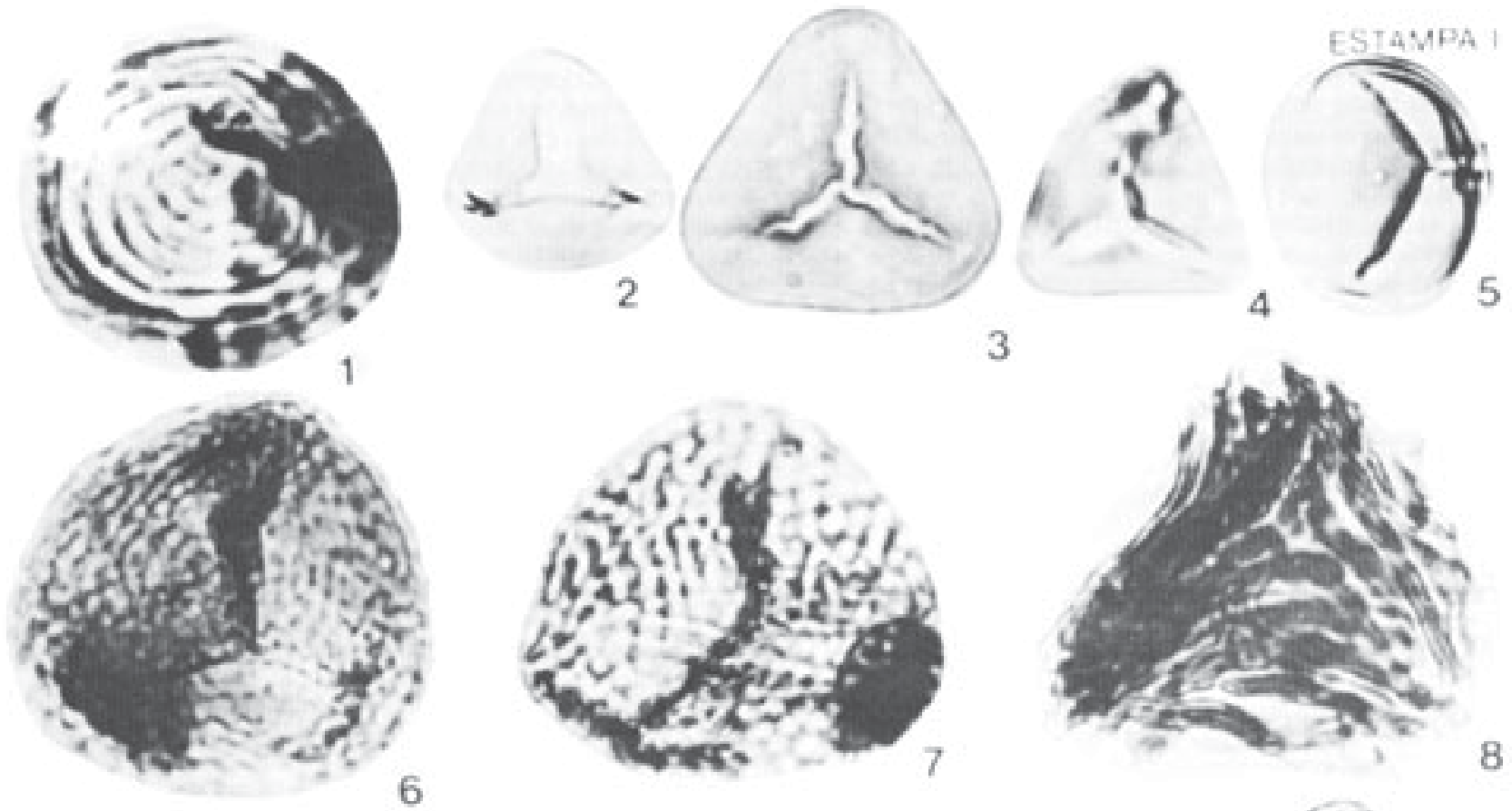

3
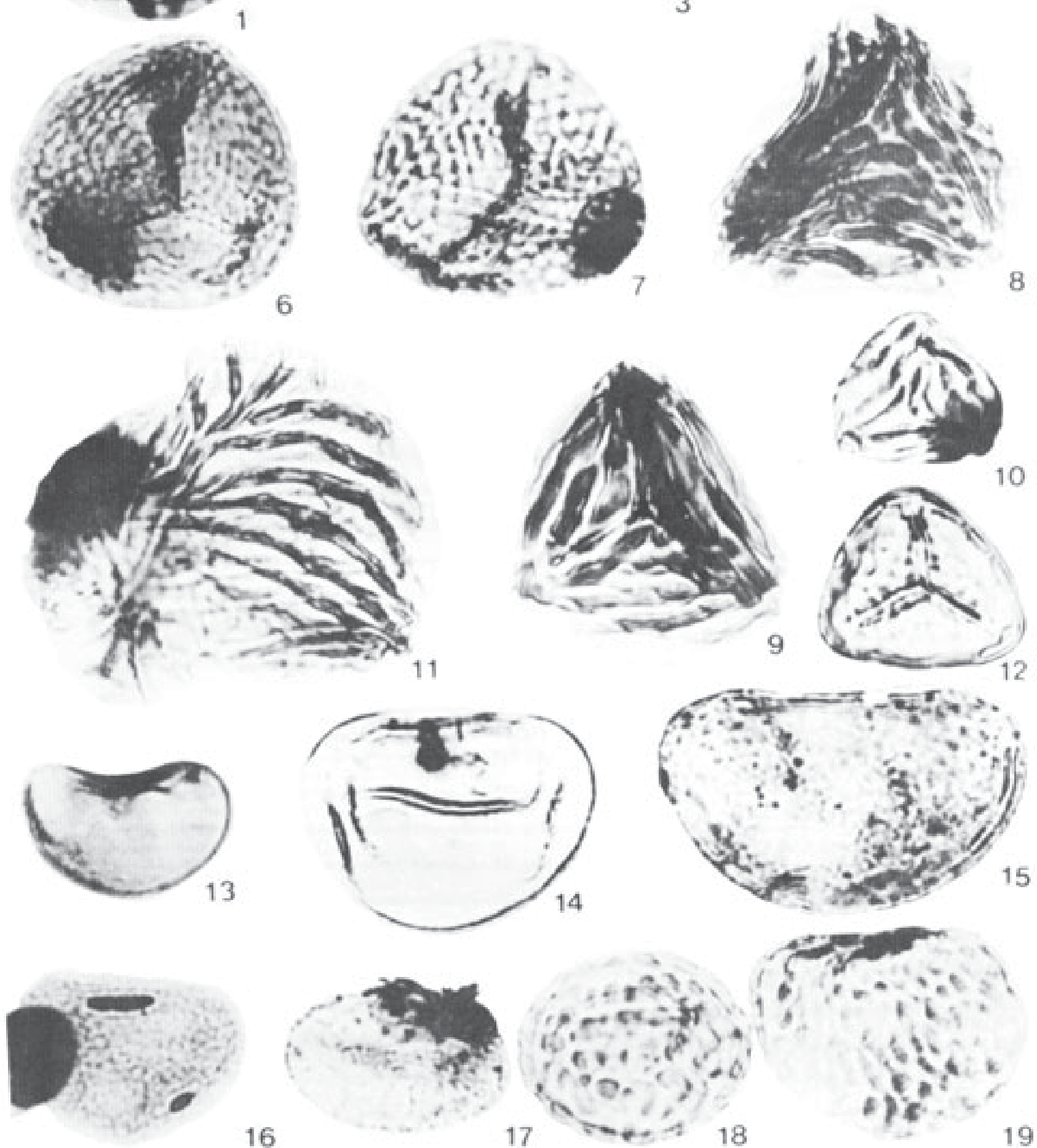


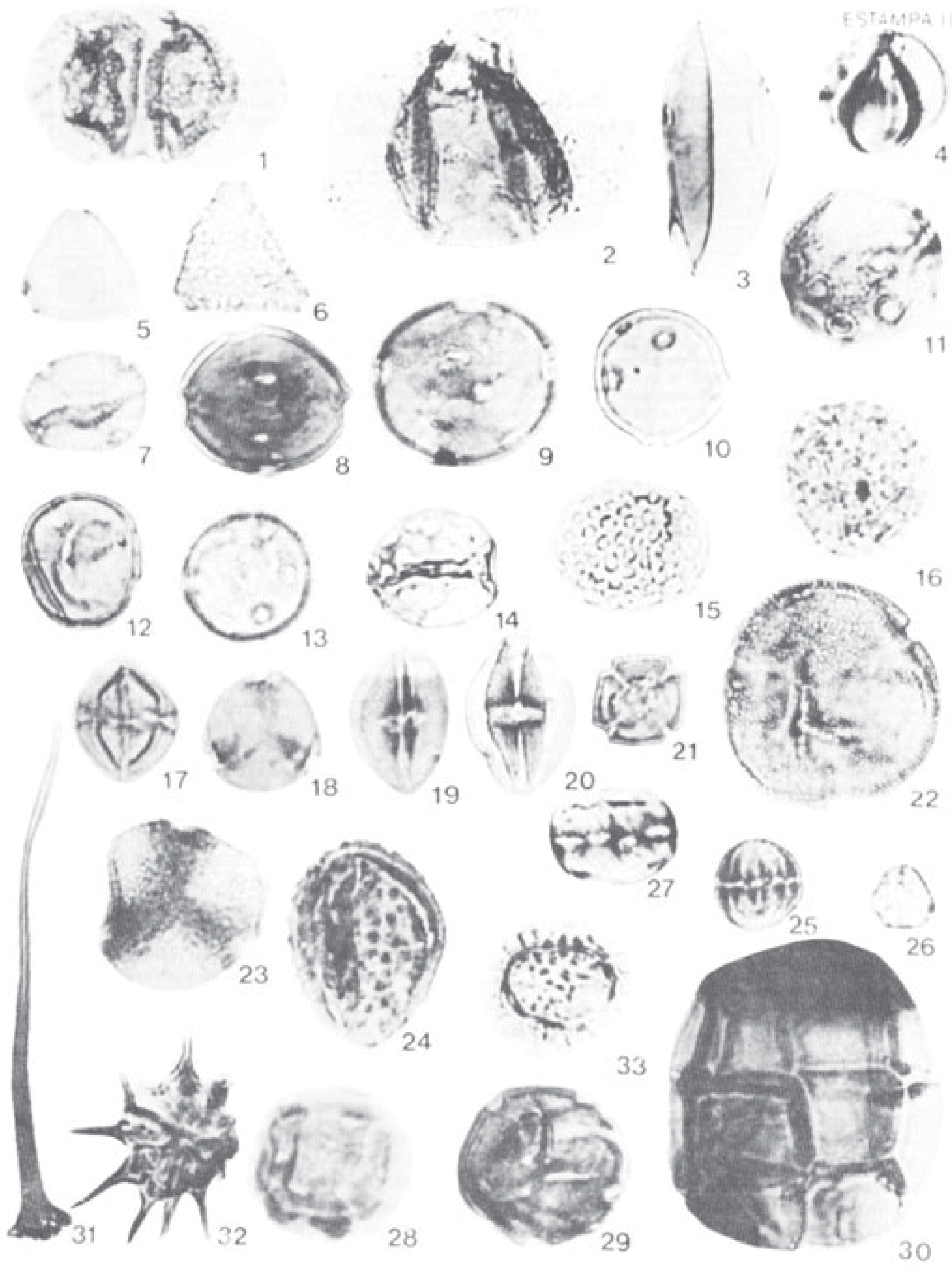

